\title{
La pizarra interactiva una estrategia metodológica de uso para apoyar la enseñanza y aprendizaje de la Matemática.[1]
}

\author{
Gonzalo Villarreal Farah \\ gvillarracomenius.usach.cl \\ Centro Comenius Universidad de Santiago de \\ chile
}

\section{Antecedentes}

\subsection{El problema}

Los resultados en las diferentes pruebas nacionales e internacionales de medición de logros de aprendizajes en matemática, son deficitarios. En particular, lo referido a la prueba nacional SIMCE y la internacional TIMSS[4], han registrado en los diferentes niveles una mantención o disminución en los puntajes.

Múltiples son los avances en materia educacional que ha estado impulsando el Ministerio de Educación de Chile. En particular el proyecto Enlaces (www.redenlaces.cl), ha permitido establecer vínculos de colaboración, trabajo y confianzas entre el Ministerio de Educación, Universidades y establecimientos educacionales, permitiendo conocer mejor la realidad del sistema educacional nacional.

Numerosas investigaciones sobre el uso de las TIC, como apoyo a los aprendizajes de los alumnos, tienen como principales conclusiones referidas a que los docentes son el aspecto central en su inserción a la escuela y la segunda que se requiere de un modelo curricular que permita en forma explicita integrar las TIC, de manera que se logren mejoras en los aprendizajes (Cabero, 2001; Cuban, 2001).

En términos generales, cabe señalar, que desde una mirada a lo que pasa en una sala de clases, es deseable que la tecnología sea integrada a tal punto que pase desapercibida, donde estudiantes y profesores no se centren en su uso, si no que la consideren como una herramienta o un medio, de apoyo a los procesos de aprendizaje del alumno (Gros, 2000).

Otro aspecto a considerar, es la forma en que profesores y alumnos están acostumbrados a trabajar, según Monereo (2000), se esta acostumbrado a un compromiso didáctico del tipo unidireccional, donde las expectativas del alumno están controladas por el profesor.

El informe UNESCO[5], al referirse a la experiencia de Enlaces, señala que por medio de observaciones realizadas en las escuelas, que la innovación surge como resultado de las prácticas pedagógicas actuales. Los docentes se encuentran más dispuestos a utilizar la tecnología si pueden relacionarla de una manera simple y directa a sus clases y a los materiales y modelos pedagógicos que utilizan normalmente.

Adicionalmente, estudios señalan que algunos obstáculos para el uso de las tecnologías en la sala de clase, se refieren, a la carencia de habilidades en los profesores para usar estos recursos, dificultad de acceso cuando se le requiere, dificultad de acceso a recursos que se vinculen significativamente con los temas tratados (Pelton \& Pelton). Las mismas autoras señalan que se alcanza un uso extenso en la 
sala de clases si esta es intuitiva, fácil de usar y productiva.

De este modo, es importante contar con soluciones curriculares, acordes con los planes y programas vigentes, con capacidad para facilitar procesos de aprendizajes pertinentes, además que haga uso efectivo de las tecnologías de la información, tienen grandes posibilidades de ser percibidas como adecuadas y necesarias en la situación actual del país.

Es así que se requiere de un modelo curricular que permita en forma explícita integrar las tecnologías, de manera que se logren mejoras en los aprendizajes. De esta manera, un aspecto interesante a estudiar y lograr, es la inserción de la tecnología a la sala de clases, en particular el que la pizarra interactiva permite tanto que profesores como alumnos, cambien sus prácticas, donde el alumno tome un rol más activo, se involucre en su aprendizaje, se generen y motiven discusiones en torno a los temas tratados, en definitiva se "hable" y "haga" matemática.

\subsection{El aprendizaje de la matemático}

Un aspecto importante en el aprendizaje matemático, lo es el conocimiento de los procedimientos de su construcción. Según Onrubia, Cochera y Barberà (2001), en estrecha relación con este conocimiento procedimental -relacionado con el saber hacer-, esta el conocimiento declarativo relacionado con el saber decir-.

Por otra parte, los estudiantes en muchas oportunidades aprenden procedimientos en forma algorítmica, sin saber necesariamente en que contexto utilizarlo (Schoenfeld, 1992). Frente a este problema, es importante, como factor de apoyo al aprendizaje de la matemática, el que los alumnos manejen el conocimiento condicional, el cual permite a un estudiante saber cuando y cómo aplicar los procedimientos aprendidos.

Wertheimer (1991), señala que aunque hay alumnos que "dominan" los hechos y procedimientos relevantes para resolver determinados problemas, no comprenden de manera significativa y críticamente importante, las ideas subyacentes en los procedimientos, siendo su dominio importante, pero no es lo único. De esta manera el poder que radica en el aprendizaje de la matemática, según Wertheimer, es la capacidad de usarla.

Para Schoenfeld (1989), la enseñanza de la matemática debe centrarse en el desarrollo del poder matemático, desarrollando aptitudes para: entender conceptos y métodos matemáticos; discernir relaciones matemáticas; razonar lógicamente; y aplicar conceptos, métodos y relaciones matemáticos para resolver una variedad de problemas no rutinarios. Para este autor, la resolución de problema solo cubre parte del "pensamiento matemático", siendo importante desarrollar habilidades metacognitivas y el desarrollo de un punto de vista matemático.

\subsection{Los recursos tecnológicos}

Una dificultad al intentar utilizar herramientas TIC en la enseñanza de la matemática, es el cambio necesario en la actuación pedagógica del profesor, ya que su uso implica un cambio de estrategia de enseñanza. Ya no es útil un esquema expositivo y lineal. Se requiere diseñar y experimentar estrategias para facilitar la interacción del alumno con los conceptos matemáticos. Así, surgen actividades como: experimentar, conjeturar, generalizar, poner a prueba hipótesis, deducir, reflexionar, etc., que son elementos extraños a una situación de clases expositiva normal.

Para organizar la forma en que la tecnología pueda tener efectos importantes en la educación de las matemáticas, Rubin (2000) propone cinco tipos de oportunidades generadas por las TIC, las cuales son: conexiones dinámicas; herramientas sofisticadas; comunidades ricas en recursos matemáticos; herramientas de diseño y construcción; y herramientas para explorar complejidad. En particular, las conexiones dinámicas: se refiere a que para la gran mayoría de los estudiantes, la matemática es a menudo algo abstracto, dificultando el aprendizaje de la matemática. La tecnología puede ayudar, al 
ser máquinas visuales, proporcionando además, medios que permiten hacer estas visualizaciones dinámicas. En este espacio, se encuentran también los simuladores, que son representaciones de la vida diaria, modeladas, que permiten a los estudiantes observar, manipular y entender el funcionamiento de situaciones reales.

Para Martín, Beltrán y Pérez (2003), trabajar con tecnología entrega muchos elementos que son esenciales en los nuevos escenarios, referidos a: ambientes realistas y enriquecidos; desarrollo del pensamiento estratégico; descubrir el problema; representación del problema; desarrollo metacognitivo; y facilitar interacciones de grupo.

El uso de las TIC, permite que los estudiantes puedan pasar de los elementos concretos a lo abstracto, pudiendo desarrollar generalizaciones de las situaciones trabajadas, aumentando sus posibilidades de adquisición de conocimientos y habilidades.

Muchos problemas requieren usar y manipular modelos, donde las TIC, además de generarlos, permiten visualizarlos y utilizar diagramas dinámicos, donde los estudiantes visualicen, manipulen y entiendan, motivándose a realizar conjeturas en forma intuitiva y posteriormente verificarlas (Baugh y Raymond, 2003).

La tecnología, ha reducido los tiempos necesarios de una clase, para dominar los algoritmos de papel y lápiz, que fueron considerados necesarios para el desempeño aritmético competente. Las tecnologías, permiten trabajar con problemas sin verse obstaculizados por realizar cálculos complejos. Las TIC permiten que exista mayor tiempo disponible en el aula para el estudio de la esencia de la matemática, pudiéndose usar como herramientas para ayudar a los estudiantes a entender las situaciones problemáticas mediante el análisis matemático (Schoenfeld, 1989).

En términos generales los recursos TIC, permiten y facilita manejar datos y su posterior manipulación pudiendo hacer uso de un gran número de herramientas, como lo son las funciones matemáticas, gráficos, inserción de distintos objetos, manipulación de objetos, manejos de mapas conceptuales, manejo de formatos, entre otros elementos. Permite disminuir el nivel de abstracción es más transparente, quedando los procedimientos expuestos y visibles, el alumno se focaliza en los aspectos importantes sin tener distracciones (Feicht, 2000; Baker y Sugden, 2003).

Cabe señalar, que en los últimos años, ha existido un uso de la tecnología principalmente como un instrumento de producción, en la cual los estudiantes usan las TIC para buscar información, hacer sus informes, usar software del tipo instruccional o tutor. Algunos autores sugiere su uso, más desde la perspectiva de la construcción cognitiva, lo que Jonassen (2000) señala "herramientas de la mente", para interpretar y organizar su conocimiento personal y Martín et al. (2003), quienes sugieren un uso de las TIC, interpretándolos como instrumentos cognitivos o instrumentos mentales, permitiendo que el estudiante construya su propio conocimiento en forma constructiva.

\subsection{La pizarra interactiva}

Esta es una tecnología que se integra a la sala de clases, abriendo un mundo de posibilidades y permitiendo ser "la punta" de la generación de innovaciones y de cambios en los roles del profesor, alumno y en la forma de trabajo. No limita, ya que puede usarse cuando se quiera, de manera que si algo no funciona, el profesor puede desarrollar su clase sin esta. Además permite aprendizajes más significativos y vinculados a la vida real. Da acceso a más recursos al profesor para modificar las estrategias metodológicas y los estudiantes se motivan e interesan más, permitiendo acceso y manejo de la información en tiempo real (Marquès, 2002).

Si bien se logra un trabajo colaborativo, es importante que los alumnos desarrollen su autonomía en el aprendizaje y construyan conocimientos significativos. Permite tener reacciones mutuas profesor alumno y en forma continua, de manera que puede modificar la sesión en la medida que la esta desarrollando, para resolver necesidades de la clase. Por medio de las preguntas y respuestas con los 
alumnos, el profesor puede dirigir el trabajo, según las reacciones de sus alumnos, pudiendo también manejar de mejor manera los avances individuales a partir de la respuesta de cada alumno.

Algunas tecnologías que han entrado al aula, se puede mencionar la incorporación de calculadoras, el computador con proyector, uno a tres computadores y las pizarras interactivas, siendo estas últimas las menos difundidas en Chile. En particular estas últimas han demostrado una facilidad de integrarse ala sala de clases, al ser un recurso natural para los docente, además de ser factible su adopción por parte de docentes y alumnos (Marquès, 2002, Villarreal, 2005). Por otra parte, las pizarras interactivas, tienen un potencial como recurso que se integra a las estrategias metodológicas que la reforma educacional chilena fomenta, además las principales ventajas de estos recursos, se refiere a que: permite una renovación tanto metodológica, como de los procesos de enseñanza y aprendizaje, además de incrementar la motivación de los estudiantes y profesores y facilita aprendizajes más significativos, vinculados al mundo real (Villarreal 2005).

\subsection{Las características generales de la pizarra interactiva}

Las pizarras utilizadas, que fueron facilitadas por la empresa Latín Telecom, quienes son distribuidores de las pizarras Smart Borrad, las que tiene conexión directa al computador; permitiendo almacenar lo que se escribe en ella; un uso de los documentos capturados (impresión, edición, envío por correo, etc.); escritura electrónica con plumones convencionales (cuatro colores); posee función Touchscreen interactiva sobre cualquier aplicación (requiere proyector), junto con poder guardar todo lo que se haga con ella incluida el sonido ambiental (voz de profesor y alumnos).

De esta manera en la sala de clases, se contó un computador, un proyector multimedia y la pizarra todos estos recursos interconectados, permitiendo desde la pizarra activar y manipular los diferentes software e Internet.

El software utilizado, fueron: PowerPoint; programas tipo applet tanto desarrollado por el Centro Comenius como algunos que se acceden por internet; recursos Excel, tanto prediseñados como uso de la planilla donde profesores y alumnos en conjunto construyeron gráficas, modelo y tablas: el software graphmatica, para desarrollar funciones y manipularlas.

\subsection{Características del modelo interactivo}

El modelo interactivo para el aprendizaje matemático, fue desarrollado en el marco del proyecto Fondef D00I1073 Aprender matemática creando soluciones, con aportes del Estado de Chile, por el Centro Comenius de la Universidad de Santiago de Chile, entre los años 2001 y 2004. Este modelo, se entiende como una formulación teórica (ideal) acerca de los elementos básicos que constituyen una situación apropiada de enseñanza y aprendizaje del conocimiento matemático y de la interrelación dinámica que existe entre dichos elementos. En su aplicación práctica, el modelo sirve como procedimiento para orientar las decisiones de quienes generan situaciones de enseñanza y aprendizaje de la matemática; de los docentes en su acción de facilitación de los aprendizajes y de quienes evalúen los aprendizajes alcanzados por los estudiantes. Consecuentemente, en su formulación se encuentran orientaciones y criterios para adoptar decisiones en relación con los diferentes momentos involucrados y, cuando corresponde, las orientaciones están referidas a los diferentes actores que participan en el proceso.

La visión más sintética que podemos ofrecer de la forma de pensar y de actuar que aquí se presenta, se acerca mucho a la expresión citada del Madison Project: "conjetura - trata, pon la idea a prueba observa lo que sucede y... aprende cómo seguir". Esto señala la búsqueda consciente de un modelo que potencie el desarrollo de un alumno independiente, que en interacción con el conocimiento y el mundo que lo rodea aprende, organiza su saber matemático como parte de su construcción como ser individual a la vez que comprometido con su entorno.

El modelo integra diferentes elementos como lo son la formación docente, los libros, los recursos y 
formación en tecnología. Se cuenta con material para el alumno (actividades, guías, proyectos, etc.), material del profesor (sugerencias para trabajar los materiales, los contenidos y las tecnologías), material de referencia (tratamiento de la matemática), recursos concretos (fichas, dados, juegos, etc.), evaluaciones y recursos tecnológicos. Se apoya con un curso semipresencial para los docentes y acompañamiento en terreno a profesores y alumnos (www.comenius.usach.cl/enlacesmat).

El núcleo del modelo se basa en la entrega de recursos y materiales, para profesores y alumnos, cambiando su rol y la forma de trabajo de la sala de clases, centrándose el proceso en el alumno. Es así que el profesor "sale de la pizarra", hace una presentación inicial del tema que trabajarán, luego los alumnos en forma individual y/o grupal, comienzan a trabajar con materiales especialmente desarrollados para cada sesión de trabajo del año académico, posteriormente el docente responde dudas a cada alumno, al grupo de trabajo o a la clase en general, según corresponda, a continuación el profesor hace un cierre "pasando en limpio" la matemática trabajada, construyendo a partir de las dudas presentadas y incentivando una participación activa de los alumnos.

La implelmentación de este modelo, en el marco del proyecto Fondef ya mensionado y poateriormente en su implementación en el proyecto Enlaces Matemática, con el Centro de Educación y Tecnología del Ministerio de Educación de Chile, ha permitido trabajar con más de 70 profesores y 2.800 alumnos del grado 10, además para el año 2006 se implementará el modelo interactivo con 100 docentes y más dee 4.000 alumnos de los grados 10 y 11, para posteriormente el 2007 implementarlo con unos 200 docentes y 8.000 alumnos de los grados 9 al 12 .

Es en este marco, que se desarrolló un proyecto con el Centro de Educación y Tecnología del Ministerio de Educación de Chile y el aporte de la empresa Latín Telecom, que hace uso de pizarras interactivas para apoyar el aprendizaje de la matemática.

\subsection{Estrategia de uso de la pizarra interactiva exploradas}

La estategia utilizada consistió en entregar diferentes recursos para potenciar y organizar las sesiones de trabajo. De esta manera se entregaron actividades y guías para los alumnos, sugerencias metodológicas a las profesoras, en las cuales se les proponía forma de trabajar determinados contenidos haciendo uso de las tecnologías. Como organizador de las clases se desarrollaron una presentación en PowerPoint para cada guía, de manera de apoyar el lanzamiento de cada tema, presentándolo, mostrando imágenes, y los objetivos a desarrollar. Durante el desarrollo de la clase la presentación apoyaba en los diferentes momentos que tenía, haciendo interactuar a los alumnos, proponiendo problemas, vinculando a recursos TIC (planillas electrónicas, graficador, applet, Internet, etc.), permitiendo un trabajo interactivo, dinámico, colaborartivo y contextualizado. De igual manera la presentación, disponía de espacios para realizar los cierres de las sesiones. De esta manera se mantenía la organización, el ritmo, la interacción de la sesión por medio de esta estrategia.

Se exploró, el uso de la pizarras interactiva, desde la perspectiva que permite su uso colectivo, haciendo explícito el conocimiento a toda la clase, tomando los alumnos un papel más activo y el profesor trabajara sin perder la visual del grupo completo e interactuara con todos o en forma individual. Es así, que la pizarra fue un espacio menos intimidador para el profesor y más familiar, resultó más cómodo, permitió manipular los diferentes software y al mismo tiempo utilizar plumones, para destacar algunos aspectos de manera natural e inmediata, esta acción permitió al estudiante facilitar su expresión, se guardaba lo que se trabajaba en la pizarra, para su posterior uso por los alumnos que la requirieran.

Se exploró desde el uso instrumental de las TIC (cálculo, organización de datos, graficación, tabulación, etc.), tendiendo a priorizar el uso como herramienta de construcción del conocimiento, donde los alumnos actuaron como diseñadores, analizaban los problemas, generaron modelos, entre otros. Así, los estudiantes, para representar lo que sabían, necesariamente se involucraron, aumentando su pensamiento crítico acerca del contenido que están estudiando, sirviendo de andamiajes a diferentes formas de razonamiento acerca del contenido, donde los estudiantes piensan 
de forma diferentes y de manera significativa respecto lo que saben.

Otro factor a observado, fue que la pizarra interactiva, redujo los tiempos necesarios de una clase, para dominar los algoritmos de papel y lápiz, que en alguna época fueron considerados necesarios para el desempeño aritmético competente. Las tecnologías, permitió a los alumnos enfrentar aplicaciones complejas, modelando y trabajar con problemas sin verse obstaculizados por la incapacidad de realizar cálculos complejos o demandante tiempo.

\section{Metodología}

\subsection{Aspectos metodológicos y didácticos}

Se esperó producir un cambio en los roles habituales de profesor y alumno. Existió una definición clara y consensuada de estos roles, donde los profesores proponían los temas de trabajo, hacían una presentación y motivación inicial, apoyados por una presentación en PowerPoint que les era entregada por el proyecto, que permitió "lanzar los temas" y posteriormente desarrollarlos y llevar el "ritmo" de la sesión y apoyar y organizar su desarrollo. A cada alumno, se le entregó materiales con actividades, para desarrollar en cada sesión. Durante la sesión, las profesoras intervenían respondiendo preguntas individuales, grupales y/o al curso, haciéndoles preguntas para guiarlos, para luego hacer un cierre en conjunto con los alumnos, donde se construía y consideraban las dudas planteadas por éstos, formalizando la matemática.

Por otra parte, los alumnos tomaron un rol activo, desarrollaron las actividades propuestas, realizaban conjeturas, probaban sus hipótesis, buscaron las soluciones a los problemas planteados, discutían, analizaban, reflexionaban, preguntaban.

En este marco la pizarra interactiva fue usada en la presentación inicial del tema tratado, en la realización de cálculos, generación de gráficos, usos de planillas, graficadores y applet, en la búsqueda y organización de la información, además de ser un instrumento que permitió apoyar las discusiones, generar reflexiones, construir modelos, analizar simulaciones presentadas y manipuladas por las profesoras y los mismos alumnos, en la búsqueda de las respuestas. Tanto alumnos como profesoras la utilizaron, indistintamente, en diferentes momentos del trabajo en la sala de clases, pasando a ser un recurso adicional.

Al ser una pizarra, fue un recurso que las profesoras pudieron encontrar más cercano, permitiéndoles manejar y tener una visual completa del curso, los alumnos se motivaron, interactuaron y participaron más, pudiendo manejar información en tiempo real.

En este trabajo, al usar la pizarra interactiva y por ende el computador, hicieron explícito su pensamiento, sus propuestas, sus modelos matemáticos, su conocimiento, además de hacer explícito el uso de los recursos tecnológicos, observándose y discutiendo las retroalimentaciones obtenidas en la utilización de estos recursos. De esta manera, uno de los principales problemas que existe en el aprendizaje de la matemática, que se refiere a su visibilidad, su dificultad en la simbología que se maneja, en lo abstracto, al utilizar esta estrategia de trabajo y según la opinión de los profesores y alumnos, se vio disminuida.

\subsection{Diseño de la experimentación}

El trabajo correspondió a una investigación cuasi experimental, con grupo de control, con pretest (diagnóstico) y postest (posprueba). Fue un cuasiexperimento, desde la perspectiva que se trabajó con grupos naturales configurados como curso, previo a la experiencia.

Se trabajó con dos establecimientos educacionales, con profesoras del área de la matemática del nivel secundaria -grado 10-, en establecimientos educacionales que participan del proyecto Enlaces del Ministerio de Educación de Chile (www.redenlaces.cl) y que durante el año 2004 participaron del 
proyecto Enlaces Matemática (www.comenius.usach.cl/enlacesmat).

Se trabajó con 4 grupos experimentales y 4 grupos controles (una profesora y un curso de aproximadamente 40 alumnos), donde cada profesora trabajó un grupo experimental y uno control. Los establecimientos fueron de la región metropolitana, de nivel socio económico medio - bajo, de alta vulnerabilidad social, un establecimientos municipalizados y otro particular subvencionado.

A cada curso se le aplicó una prueba de diagnóstico que permitió medir los contenidos en el tema a tratar, luego en el experimental se trabajo con la metodología propuesta y en el control las profesoras trabajaron como lo hacen habitualmente, en particular en su sala de clases sin apoyo de TIC. Al término de la unidad propuesta se aplicó el postest, de manera de comparar los aprendizajes alcanzados por cada grupo. Tanto los grupos experimentales como control, hicieron uso del modelo interactivo, variando solo la inclusión de la pizarra interactiva en las salas del grupo experimental.

Adicionalmente, se aplicó a cada grupo un test de actitudes, antes de iniciado el proceso y al término de la realización de la experiencia. Se aplicó una pauta de observación, para obtener información respecto al comportamiento del profesor, alumno, uso de los recursos tecnológicos y estado de la sala de clases. Además se desarrollaron dos pruebas parciales que midieron los avances en las unidades, realizadas tanto a los grupos experimental como control, junto con implementar un cuestionario a los alumnos y profesores de las salas experimentales.

El trabajo en los establecimientos, tuvo una duración aproximada, del tratamiento de los contenidos y uso de la pizarra interactiva, de dos meses para los cursos experimentales y similar tiempo para los grupos controles, estos últimos sin uso de tecnología.

\section{Resultados}

\subsection{Análisis cuantitativos}

El proyecto desarrolló una prueba de diagnóstico y una prueba final. Los resultados de la prueba de diagnóstico aplicada a 313 alumnos y la prueba final aplicada a 306 alumnos, que participaron del proyecto, se encuentran en la siguiente tabla.

Tabla 1: resultados de prueba de diagnóstico y final por tipo de sala

\begin{tabular}{lcccc}
\multicolumn{1}{c}{ Tipo } & \multicolumn{2}{c}{ Diagnóstico } & \multicolumn{2}{c}{ Final } \\
& Pr C & $\mathbf{N}^{\mathbf{0}}$ alumnos & Pr C & $\mathbf{N}^{\mathbf{0}}$ alumnos \\
Experimental & 27,5 & 160 & 68,3 & 157 \\
Control & 30,1 & 153 & 64,8 & 149 \\
Diferencia & $\mathbf{2 , 6}$ & & $\mathbf{3 , 5}$ &
\end{tabular}

Los resultados del grupo experimental, fueron inferiores a los del grupo control, teniendo una variación de $10 \%$, con una diferencia de 2,6 puntos porcentuales, donde la diferencia no es significativa. Respecto a la prueba final, los resultados del grupo experimenta fueron superiores a los del grupo control, con una variación porcentual de $5 \%$, con una diferencia de 3,5 puntos porcentuales, no siendo significativa. Se destaca que el curso experimental aumentó en 40,8 puntos porcentuales, entre la prueba de diagnóstico y la final, en comparación con la diferencia alcanzada por el grupo control correspondiendo a 34,7 puntos porcentuales, con 6,1 puntos porcentuales, un 17,6\% superior del grupo experimental al control.

\subsubsection{Resultado por distribución de datos}

Se agruparon los datos en intervalos de frecuencia (categorizar en 6 intervalos de amplitud 16 puntos porcentuales cada uno) adecuados al número de datos trabajados, de tal manera de poder apreciar que cantidad de alumnos se distribuye en cada intervalo. 
De esta manera, los intervalos quedaron:

\section{Categorías Porcentaje}

$$
\begin{aligned}
& \text { 1: } 0-16,0000 \\
& \text { 2: } 16,0001-32,0000 \\
& \text { 3: } 32,0001-48,0000 \\
& \text { 4: } 48,0001-64,0000 \\
& \text { 5: } 64,0001-80,0000 \\
& \text { 6: } 80,0001 \mathrm{y}+
\end{aligned}
$$

Los gráficos 1 y 2 presentan la distribución de los resultados de la prueba de diagnóstico para los grupos experimental y control
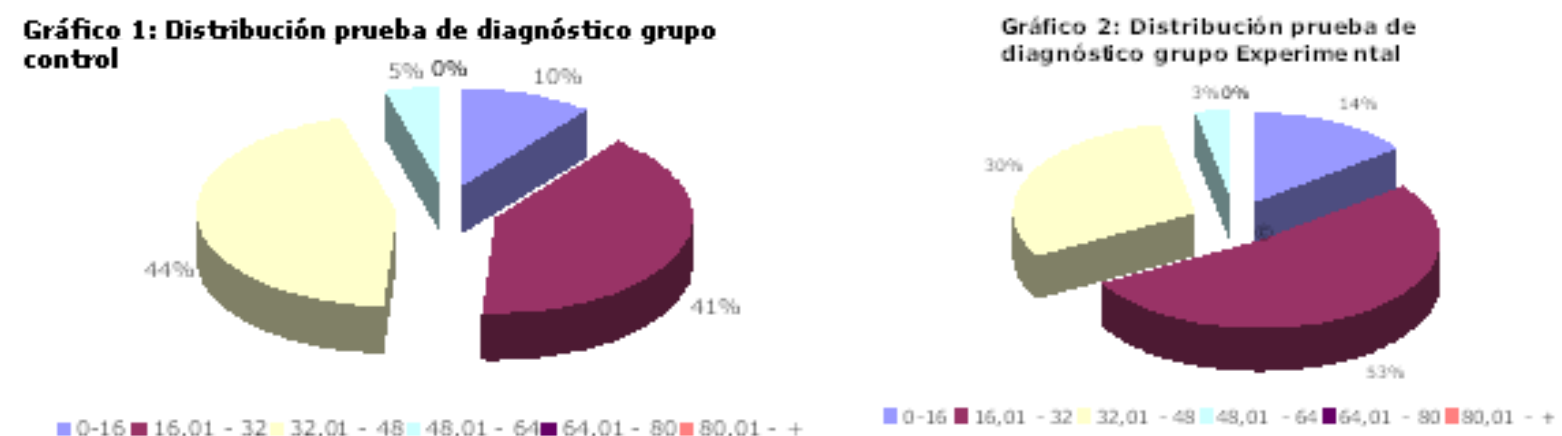

Se puede observar que ambos grupos no tienen alumnos con porcentajes de logros superiores a $64 \%$. Adsemás el mayor número de alum nos se encuentra con resultados entreel 16,01 al 48 porciento de logros, siendo mejores los resultados del grupo experimental al tener $14 \%$ más de alumnos en el rango $32,01-48$.

Los gráficos 3 y 4 muestran la distribución de la Prueba final:
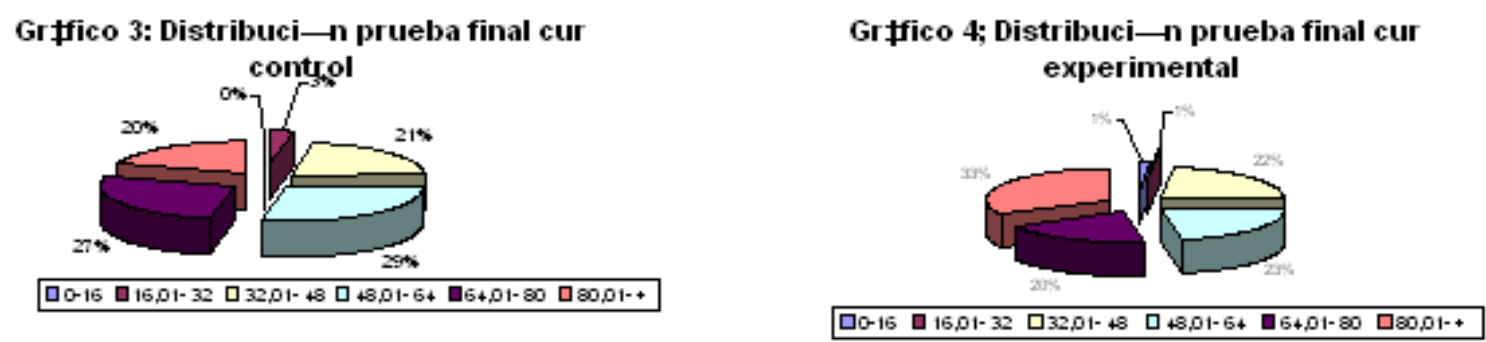

Los gráficos anteriores muestran que los alumnos del grupo control tiene $13 \%$ más de alumnos que el grupo experimental en el rango 48,1 al 80 porciento de logros, sin embargo el grupo experimental tiene un $13 \%$ más de alumnos que el grupo control en el rango mayores a 80,01 porciento de logros.

\subsubsection{Prueba parciales $N^{o} 1$ y $N^{o} 2$}

Se desarrollaron dos pruebas de avance de unidad; la evaluación $\mathrm{N}^{\mathrm{o}} 1$ de Variables y modelos matemáticos, aplicada a 326 alumnos y la evaluación $N^{\circ} 2$ de Funciones lineales, aplicada a 311 alumnos. Los resultados de ambas pruebas, se encuentran en la tabla que se presenta a continuación. 


\begin{tabular}{lcccc} 
Tipo & $\begin{array}{c}\text { Prueba parcial } \mathbf{N}^{\mathbf{0}} \mathbf{1} \\
\text { PR C }\end{array}$ & \multicolumn{2}{c}{$\begin{array}{c}\text { Prueba parcial } \\
\text { No }\end{array}$} \\
Experimental & alumnos & PR C & $\mathbf{N}^{\mathbf{0}}$ alumnos \\
Control & 67,4 & 169 & 55,2 & 166 \\
Diferencia & 62,6 & 157 & 46,6 & 145 \\
& $\mathbf{4 , 8}$ & 326 & $\mathbf{8 , 7}$ & 311
\end{tabular}

Se puede observar que los resultados de los alumnos que participaron en el proyecto fueron superiores a los del grupo control en ambas pruebas parciales, teniendo una variación porcentual de $8 \%$ en la prueba $\mathrm{N}^{\mathrm{o}} 1$ y de $19 \%$ en la prueba $\mathrm{N}^{\circ} 2$; con una diferencia de 4,8 y 8,7 puntos porcentuales respectivamente.

La tabla 3, presenta los resultados para la prueba final de los grupos control y experimental.

Tabla 3: resultados de prueba final comparando grupo control y experimental

$\begin{array}{lccccc}\quad \text { Prueba final } & \text { Promedio } & \text { N } & \text { Desv. Est. } & \text { P-valor } & \text { Resultado test } \\ \text { Control } & 65 & 150 & 18 & 0.09600 & \text { La diferencia no es } \\ \text { Experimental } & 69 & 157 & 21 & & \text { significativa }\end{array}$

*No se asume igualdad de varianza entre los grupos

Test "T" de comparación de medias para muestras independientes

Si bien la diferencia en resultados existente entre las pruebas inicial y final es estadísticamente significativa, la diferencia existente entre el grupo control y experimental en la prueba final no es estadísticamente significativa.

\subsubsection{Test de actitudes}

El tes utilizado fue una adaptación del Test de Actitud hacia el estudio de la matemática de Fennema y Sherman, éste fue desarrollado por Elizabeth Fennema y Julia Sherman, bajo los auspicios de la "Nacional Science Foundation".

El test fue aplicado en dos oportunidades a dos grupos de alumnos, uno experimental y uno control, compuestos por 4 cursos, tres pertenecientes al Colegio Santa María de Santiago y uno del Colegio Benjamín Franklin, con un total de 158 alumnos en el grupo control y 161 en el grupo experimental.

La tabla 4 presenta los resultados del pretest y postest de actitud para grupo control y experimental

\begin{tabular}{|c|c|c|c|c|}
\hline \multirow{2}{*}{ Preguntas } & \multicolumn{2}{|c|}{ Pretest } & \multicolumn{2}{|c|}{ Postest } \\
\hline & Control & Experimental & Control & Experimental \\
\hline $\begin{array}{l}\text { Confianza personal } \\
\text { sobre el tema }\end{array}$ & 42 & 43 & 41 & 41 \\
\hline Utilidad del contenido & 45 & 47 & 43 & 43 \\
\hline Actitud hara el éxito & 43 & 45 & 43 & 43 \\
\hline $\begin{array}{ll}\text { Opinión } & \text { de los } \\
\text { profesores } & \end{array}$ & 45 & 45 & 42 & 41 \\
\hline Total & 175 & 179 & 169 & 168 \\
\hline
\end{tabular}

En grupo experimental, en el pretest, todas las dimensiones son superiores a los 43 (de un máximo de 60) puntos en promedio, siendo superior al control. Para el grupo control, en la aplicación del post test, en cada dimensión, se obtuvo por sobre el 41, obteniendo el grupo experimental valores muy similares al grupo control.

Se puede observar que el pre test del grupo experimental es superior en 4 puntos, sin embargo la diferencia en el postest dentro de ambos grupos es 1 a favor del grupo control. Además se observa una leve disminución, para ambos grupos, entre el pretest y el postest a favor del primero. 
Se puede señalar que tanto en el pretest como en el postest, los alumnos tienen una opinión entre indiferencia y positiva (pero no fuertemente). Posibles explicaciones de esta situación, puede ser el escaso tiempo transcurrido entre la aplicación de uno y otro test o el que ambos grupos tuviesen inicialmmente buena actitud hacia la matemática.

\subsection{Análisis cualitativo}

\subsubsection{Cuestionario Alumnos}

Al finalizar el proyecto se aplicó un cuestionario a 124 alumnos de las cuatro salas experimentales de ambos establecimientos. Este instrumento consta de un total de 49 preguntas cerradas y dos preguntas abiertas, que tuvo como objetivo evaluar aspectos referentes a las sesiones de trabajo con la pizarra interactiva, y de esta manera, obtener la visión "promedio" que tienen los alumnos de su trabajo y el de la profesora a cargo.

A continuación se presenta la tabla 5 con una descripción de los cuatro aspectos evaluados a través de este cuestionario[6].

Tabla 5: Descripción de aspectos evaluados

\begin{tabular}{|l|l|}
\hline \multicolumn{1}{|c|}{\begin{tabular}{c}
\multicolumn{1}{|c|}{ Ítem } \\
Cuestionario
\end{tabular}} & \multicolumn{1}{c|}{ Descripción } \\
\hline $\begin{array}{l}\text { Nivel de atención, } \\
\text { motivación y asistencia }\end{array}$ & $\begin{array}{l}\text { Evaluar los niveles de motivación, autoestima, actitud, respuesta, } \\
\text { participación, etc. de los alumnos. }\end{array}$ \\
\hline Aspectos generales & $\begin{array}{l}\text { Evaluar la conexión existente entre el aprendizaje y la participación } \\
\text { del alumno }\end{array}$ \\
\hline $\begin{array}{l}\text { Usos de la Pizarra por el } \\
\text { profesor }\end{array}$ & $\begin{array}{l}\text { Evaluar el uso de la pizarra por el profesor durante la aplicación del } \\
\text { proyecto }\end{array}$ \\
\hline
\end{tabular}

La tabla 6 presenta los resultados de los aspectos, que en promedio son más valorados y menos valorados por los estudiantes.

Tabla 6: Promedios por ítem / aspectos más y menos valorados

\begin{tabular}{|c|c|c|c|c|}
\hline \multirow{6}{*}{$\begin{array}{c}\text { Ítem } \\
\text { Cuestionario } \\
\text { Nivel de } \\
\text { atención, } \\
\text { motivación y } \\
\text { asistencia } \\
\text { Aspectos } \\
\text { generales }\end{array}$} & \multirow[t]{2}{*}{$\begin{array}{l}\text { Prom. } \\
\text { ítem }\end{array}$} & valoración & $\begin{array}{l}\text { Descripción } \\
\text { pregunta }\end{array}$ & $\begin{array}{l}\text { Prom. } \\
\text { Preg. }\end{array}$ \\
\hline & & más & Facilita la organización de contenidos matemáticos & 3,1 \\
\hline & & valorados & Permitió reforzar los aprendizajes & 3,0 \\
\hline & 2,1 & menos & Disminuyó la distracción de los alumnos & 2,3 \\
\hline & & valorados & Mejoró la autoestima de los alumnos & 2,3 \\
\hline & & $\begin{array}{l}\text { más } \\
\text { valorados }\end{array}$ & $\begin{array}{l}\text { Permitió a la profesora dedicarse en los avances y } \\
\text { respuestas de los alumnos }\end{array}$ & 3,2 \\
\hline & 2,9 & & $\begin{array}{l}\text { Permitió usar recursos de la tecnología de } \\
\text { información y comunicación (TIC) }\end{array}$ & 3,2 \\
\hline & & $\begin{array}{l}\text { menos } \\
\text { valorados }\end{array}$ & $\begin{array}{l}\text { Facilitó el logro de habilidades y competencias } \\
\text { matemáticas }\end{array}$ & 2,7 \\
\hline & & & Permitió fomentar el debate & 2,5 \\
\hline Uso de las TICs & & $\begin{array}{l}\text { más } \\
\text { valorados }\end{array}$ & $\begin{array}{l}\text { Facilitó la presentación visual de contenidos } \\
\text { matemáticos }\end{array}$ & 3,2 \\
\hline & & & $\begin{array}{l}\text { Facilita trabajar diagramas, tablas, figuras y/o } \\
\text { gráficos }\end{array}$ & 3,2 \\
\hline & 3,0 & $\begin{array}{l}\text { menos } \\
\text { valorados }\end{array}$ & $\begin{array}{l}\text { Ayudaron a enfrentar situaciones complejas, reales } \\
\text { de la vida }\end{array}$ & 2,6 \\
\hline & & & Facilita trabajar simulaciones, videos y/o & \\
\hline
\end{tabular}


Usos de la

Pizarra por el profesor más val. 3,5

El profesor hace un uso de la pizarra para introducir el tema de la sesión

menos val. El profesor hace un uso de la pizarra para cerrar la sesión

En la tabla se observa que el ítem promedio mejor valorado es "Usos de la pizarra por el profesor", en éste los alumnos dan cuenta que el docente a cargo, utiliza la pizarra interactiva, tanto para introducir el tema de la sesión, como para el cierre de ésta. Así también podemos apreciar que el aspecto menos valorado es el "Nivel de atención, motivación y asistencia" que muestran los alumnos durante la aplicación del proyecto.

\subsubsection{Resultados generales preguntas abiertas}

A continuación se presentan los resultados de la opinión de los estudiantes respecto a cuáles son los beneficios y dificultades de trabajar con la pizarra electrónica.

Tabla 7: Frecuencia de aspectos más destacados

\section{Aspectos destacados}

La clase se hace más interactiva y dinámica

Facilita el proceso de Enseñanza- Aprendizaje facilitando el entendimiento y trabajo de nuevos contenidos

La profesora enseña de manera más rápida y fácil

Se trabajan los contenidos de manera visual con ejemplos gráficos, presentaciones en PowerPoint, etc.

Tabla 8: Frecuencia de aspectos menos destacados

\section{Aspectos menos destacados}

La pizarra se descalibra con facilidad retrasando la clase y provocando

distracción

Dificultades en la utilización de la pizarra

Lentitud para utilizar los programas debido a la antigüedad de los PC y falta de asistencia técnica

Desorden constante de los alumnos

\section{Frecuencia}

40

33

30

18

\section{Frecuencia}

Cabe señalar, que por el escaso tiempo en la implementación del proyecto y por tener una duración limitada, se instaló una pizarra con pedestal (no fija), el computador y proyector al frente de la pizrra, lo que tenía diferentes dificultades como son el movimiento de estos cuando eran pasados a llevar o el que hiciera sombra en la pizarra cuando una persona trabajando en esta se colocaba entre el proyector y la pizarra. Claramente estos son temas que son factibles de solucionar, dejando una pizarra fiija en lo posible, un proyector ubicado en el techo de la sala y el computador ubicarlo en un costado de la pizarra.

\subsubsection{Cuestionario Profesores}

Al finalizar el proyecto se aplicó un cuestionario a las cuatro docentes a cargo de las salas experimentales de ambos establecimientos. Este instrumento consta de un total de 16 preguntas, y tuvo como objetivo evaluar aspectos referentes a las sesiones de trabajo con la pizarra interactiva.

En relación a los cuestionarios aplicados a docentes se puede destacar que el 50\% señala que existió una "mejora significativa" y un 50\% señala que existió "cierta mejora", tanto en su trabajo como en el progreso, actitud y nivel de expectativas de los alumnos. 
En la misma encuesta, las profesoras señalan que en relación al rol interactivo asumido, un $25 \%$ indica que fue "muy superior" y un $75 \%$ que fue "superior". Las docentes señalaron que esto se atribuía al hecho de no requerir dictar contenidos a los alumnos y a disponer de software que facilitan la ejemplificación.

Adicionalmente las docentes destacaron:

El aumento de la participación en clases de aquellos alumnos que no obtenían buenos resultados antes de la aplicación del proyecto.

- El acceso a nuevas tecnologías de información y comunicación a estudiantes que no cuentan con esta posibilidad fuera del aula.

El aumento de alumnos que se interesan y motivan por indagar información que refuerce los conocimientos entregados en la sala de clases.

El aumento de alumnos que se interesa por aprender el uso de software y de otros aspectos relacionados con la pizarra en si.

\subsection{Observaciones de sesiones de trabajo}

Durante la aplicación del proyecto se programaron observaciones al 50\% de las sesiones de trabajo a dos salas experimentales, correspondiendo a una sala de cada establecimiento. La finalidad de estas observaciones fue tener un registro de lo que ocurría en la sala de clases con los alumnos, la profesora, el uso de la pizarra electrónica y su apoyo en el proceso de enseñanza y aprendizaje de la matemática.

La pauta de observación que se utilizó consta de 119 subítemes y considera las siguientes categorías: docente, sala de clases, alumnos y uso de TIC[7]. Así también, la pauta tiene espacios para observaciones abiertas, para cada categoría señalado y dos descripciones que versan sobre los recursos TIC utilizados por el profesor y por el alumno.

A continuación se presenta una tabla resumen que tiene como objeto describir y explicar las cuatro categorías evaluadas a través de la pauta de observación.

Tabla 8: descripción de categorías de pauta de observación

\begin{tabular}{|l|l|}
\hline Categoría & \multicolumn{1}{|c|}{ Descripción } \\
\hline Docente & $\begin{array}{l}\text { Evaluar los distintos aspectos pedagógicos tales como: actuación y acciones del } \\
\text { profesor, estrategias metodológicas, apertura y motivación de la sesión, desarrollo y } \\
\text { cierre de ésta, sugerencias del profesor para resolver problemas, etc. }\end{array}$ \\
\hline $\begin{array}{l}\text { Sala de } \\
\text { clases }\end{array}$ & $\begin{array}{l}\text { Evaluar las condiciones de la sala de clases, la organización de los grupos de trabajo } \\
\text { por parte de los alumnos, el trabajo al interior del grupo y entre grupos distintos, } \\
\text { recursos, clima de la sala de clases y relación profesor- alumno, etc. }\end{array}$ \\
\hline Alumnos & $\begin{array}{l}\text { Evaluar el conocimiento del contenido específico y aspectos generales tales como: } \\
\text { interés en la clase, disposición positiva con las actividades propuestas, colaboración } \\
\text { con el docente y los demás alumnos, formulación de preguntas, respeto por la } \\
\text { autonomía, etc. }\end{array}$ \\
\hline Uso de TIC & $\begin{array}{l}\text { Evaluar aspectos generales como el uso de la pizarra por parte del profesor y de los } \\
\text { alumnos, distracción de los alumnos, optimización de tiempos, etc. Evaluar el uso } \\
\text { instrumental y cognitivo de las TIC por parte de los alumnos y el docente, etc. }\end{array}$ \\
\hline
\end{tabular}

A continuación se presenta un promedio por cada categoría evaluado por las observadoras en ambos establecimientos.

Tabla 9: Promedio de resultados de observaciones por categoría 


\begin{tabular}{|c|c|c|c|}
\hline Categoría & Sub-ítems & Promedio colegio 1 & Promedio colegio 2 \\
\hline Docente & aspectos pedagógicos & 3 & 3 \\
\hline $\begin{array}{l}\text { La sala de } \\
\text { clases }\end{array}$ & La sala de clases & 3 & 3 \\
\hline \multirow[t]{2}{*}{ Alumnos } & aspectos generales & 3 & 3 \\
\hline & $\begin{array}{l}\text { conocimiento del } \\
\text { contenido específico }\end{array}$ & 2 & 4 \\
\hline \multirow{4}{*}{$\begin{array}{l}\text { Uso de las } \\
\text { TIC }\end{array}$} & Aspectos generales & 3 & 3 \\
\hline & A nivel cognitivo & 3 & 4 \\
\hline & A nivel instrumental & 3 & 4 \\
\hline & Por parte del profesor & 4 & 4 \\
\hline \multicolumn{2}{|c|}{ Promedio general } & $\overline{3}$ & 3 \\
\hline
\end{tabular}

\section{Conclusiones}

La posibilidad de realizar un proyecto de innovación, que hiciera uso de recursos tecnológicos, en particular de una pizarra interactiva, permitió alcanzar diferentes conocimientos y logros respecto a su implementación en salas de clases del nivel secundario, de dos establecimientos educacionales de la región metropolitana. A partir de esta experiencia, a continuación se presentan las principales conclusiones respecto de las hipótesis planteadas en la presentación del proyecto:

Las hipótesis planteadas, respecto a un mayor logro de aprendizajes por parte del grupo experimental en relación al grupo control, se puede señalar que en la prueba de diagnóstico, el grupo control alcanza mejores resultados que el experimental, por 3 puntos porcentuales, mientras que en las pruebas de avance de unidad uno, dos y prueba final, en promedio, los cursos experimentales alcanzan mejores logros que los de control en $4.8,8.7$ y 3.5 puntos porcentuales respectivamente.

Respecto a la prueba final, los resultados del grupo experimenta fueron superiores a los del grupo control, con una variación porcentual de $5 \%$, con una diferencia de 3,5 puntos porcentuales, no siendo significativa.

Se destaca además que el grupo experimental superó en 13\% los logros del grupo control, en el rango superior de resultados mayores a $80 \%$. Principalmente, esta diferencia corresponde a un aumento del logro de aprendizajes de los alumnos del grupo experimental que se encuentran en los rangos entre $48,01 \%$ a $80 \%$ de logros, quienes pasan al rango mayor a $80 \%$ de logros.

La segunda hipótesis, referida a un mejor actitud hacia la matemática por parte del grupo experimental en relación al grupo control, se puede indicar que en el pretest de actitud ambos grupos, tuvieron una actitud positiva hacia la matemática, con un promedio sobre el $70 \%$, sin embargo existió en el postest un leve descenso para ambos grupos, llegando al promedio de $70 \%$, teniendo igualmente, ambos grupos, una actitud positiva hacia la matemática. Algunas posibles causas de este descenso pueden ser el poco tiempo existente entre la aplicación del pre y postest y/o en que ambos grupos inicialmente tenían una positiva actitud hacia la matemática en un porcentaje complejo de superar.

Otras conclusiones:

El proyecto permitió generar conocimiento, en particular poder desarrollar una estrategia de uso, junto con detectar condiciones necesarias para una mejor implementación de estas herramientas.

La pizarra interactiva, hace más explícita la matemática y el uso de las tecnologías, para los alumnos. Efectivamente la tecnología "obliga" el ingreso de instrucciones o elementos precisos, donde se tenían retroalimentaciones inmediatas, esto junto a la posibilidad de presentar animaciones, modelos visibles, utilización de graficadores, entre otros recursos, potenció este aspecto en el aprendizaje de los alumnos. 
Que los alumnos y profesoras tuviesen guías y presentaciones previamente desarrolladas, les permitió concentrarse en los procesos de aprendizaje y en los aspectos pedagógicos respectivamente, los primeros pudieron tener un rol más interactivo, participativo, generar ideas y tener juicios críticos. A los docentes les permitió incentivar las discusiones, observar los avances de sus alumnos, detectar dificultades, responder dudas individuales, a grupos y al curso, entre otros aspectos.

Respecto a la apropiación por parte de los profesores y alumnos, del uso de la pizarra, se debe señalar que es fundamental la realización de una formación inicial de docentes, respecto a su uso, potencialidades y principalmente su uso como apoyo a la enseñanza de la matemática. El proyecto demuestra que los profesores y alumnos, si bien al principio la usan con cautela, a los dos meses han logrado una apropiación de esta herramienta. Lo anterior permite señalar que este tipo de tecnologías, son factibles de ser insertadas en establecimientos educacionales, como apoyo a los procesos educativos.

Referido al uso de las tecnologías en el proceso de enseñanza aprendizaje, se pudo observar que si bien es una tecnología que produce distracciones iniciales, en corto plazo pasa a ser un recurso adicional, especial y potente, donde la pizarra en si no es el centro del proceso, sino lo que se hace con ella, pasando a ser una tecnología "invisible".

Fue posible incorporar este tipo de tecnologías en establecimientos educacionales municipalizados y subvencionados, los profesores y alumnos la valoran y son capaces de apropiarse de ella.

\section{Bibliografía}

ABRAMOVICH, S. \& BROUWER, P. (2003). Revealing hidden mathematics curriculum to preteachers using technology: the case of partitions. International Journal of Mathematical Education in Science and Technology, 34(1), pp. 81-94.

BAUGH, I., \& RAYMOND. A. (2003). Making Math Success Happen: The Best of Learning \& Leading with Technology on Mathematics. EE.UU. ISTE.

BAKER, J. y SUGDEN, S. (2003). Spreadsheets in Education-The First 25 Years. Spreadsheets in Education. 1 (1). En http://www.sie.bond.edu.au/

CABERO, J. (2001). Tecnología Educativa. Diseño y utilización de medios en la enseñanza. Barcelona: Paidós.

CASTELLS, M. (2002). Lección inaugural del programa de doctorado sobre la sociedad de la información y del conocimiento, Documento presentado en Inauguración doctorado sociedad de la Información, Barcelona: Editorial UOC. Disponible en http://www.uoc.edu/web/esp/articles/castells/menu10.html

CUBAN, L (2001). Oversold and Underuse: Computer in the Classroom. London: Harvard University Press.

FEICHT, L. (2000). Guess and Check: A viable problem-solving strategy. Learning \& Leading with Technology, 27 ( 5), 50 - 54.

GOLDENBERG, P. (2000). Thinking (And Talking) About Technologyin Math Classrooms. En Education Development Center, Inc. http://www2.edc.org/mcc/iss tech.pdf

GROS, B. (2000). El ordenador invisible: hacia la apropiación del ordenador en la enseñanza. Barcelona: Gedisa

HEPP, P. (2003). La educación digital. En Hevia, R. (2003). La educación en Chile, hoy. Chile: 
Ediciones Universidad Diego Portales.

INFORME COCKCROFT. (1985). Las matemáticas sí cuentan. Madrid: MEC.

OCDE. (2004). Revisión de políticas nacionales de educación, Chile. Paris: OCDE.

ONRUBIA, J., COCHERA, M., y BARBERÀ, E. (2001). La enseñanza y el aprendizaje de las matemáticas: una perspectiva psicológica. En Coll, C. Palacios, J. y Marchesi, A. Desarrollo psicológico y educación. Psicología de la educación escolar. Madrid: Alianza.

MARQUÈS, P. y CASALS, P. (2002). La pizarra digital en el aula de clase,Una de las tres bases tecnológicas de la escuela del futuro. http://www.cica.es/aliens/revfuentes/num4/monografico_1.htm [consultado 12/2004]

MARQUÈS, P. (2002). La magia de la "pizarra electrónica". Una innovación al alcance de todos. Presentado en el congreso TIEC-2002, Barcelona.

MARTín, J., BELTRÁN, J. y PÉREZ, L. (2003). Como aprender con internet. Madrid: Foro pedagógico de internet.

MONEREO, C. (2000). Estrategias de aprendizaje. Madrid: Visor.

NACIONES UNIDAS (1998). Informe Anual para el Desarrollo Humano, Consumo para el desarrollo. Nueva York: Ediciones Mundi-Prensa.

PELTON, W. y PELTON, L. The Electronic Slate: Including Preservice Teachers in Research and Development. En http://web.uvic.ca/ tpelton/elslatesite.htm [consulta 12/2004]

RUBIN, A. (2000). Technology meets math education: Envisioning a practical future forum on the future of technology in education. En http://www.air-dc.org/forum/abRubin.htm

SEDICI. (2000). Métrica de la Sociedad de la Información.

SCHOENFELD, A. (1989). La enseñanza del pensamiento matemático y la resolución de problemas. En Resnick, L. y Klopfer, L. (1989). Curriculum y Cognición .Buenos Aires: Aique.

SCHOENFELD, A. (1992). Learning to think mathematically: problem solving, metacognition, and sense-making in mathematics. En Handbook for Research on Mathematics Teaching and Learning ( pp. 334-370). New York: MacMillan.

SILVA, J. y VILLARREAL, G. (2004). El uso de graficadores y procesadores geométricos en la enseñanza de la matemática en el nivel secundario, Marco teórico. Material desarrollado para Enlaces en Red, del Proyecto Enlaces, Mineduc.

SITES M1 (2002). Second Information Technology in Education Study. Estudio Internacional Tecnologías de Información en el Sistema Escolar, el caso de Chile. Ministerio de Educación de Chile.

UNESCO. (2000) Informe Mundial sobre la Comunicación y la Información 1999-2000.

VILLARREAL, G. (2005). Uso de la pizarra interactiva en salas de clases como apoyo a la enseñanza y aprendizaje de la matemática. Compilador Sánches Jaime $X$ Taller Internacional de Software Educativo.

VILLARREAL, G. (2004). Generación de material didáctico en Excel, Marco Teórico. Material desarrollado para Enlaces en Red, del Proyecto Enlaces, Mineduc. 
YABAR, J. M. \& ESTEVE, J. (1996). Integración curricular de los recursos tecnológicos en el área de matemáticas. En Gallego, D. J., Alonso, C. M. y Cantón, I. (Coord), Integración curricular de los recursos tecnológicos (2a. ed., pp. 129-180). Barcelona: Oikos-tau.

Notas:

[1] Este proyecto contó con la valiosa cooperación de la empresa Latín Telecom, quienes son distribuidores de las pizarras smart borrad y quienes facilitaron las pizarras interactivas utilizadas.

[2] La pizarra interactiva es una pizarra en la cual se proyectan las aplicaciones de un computador, permitiendo manejar, por medio de touchscreen, los diferentes software además de plumones y destacadores desde la pizarra.

[3] El modelo interactivo para el aprendizaje matemático, fue desarrollado en el marco del proyecto Fondef D00I1073 Aprender matemática creando soluciones, con aportes del Estado de Chile, por el Centro Comenius de la Universidad de Santiago de Chile (www.comenius.usach.cl/enlacesmat).

[4] Third International Mathematics and Science Study, donde se aplicó una prueba de matemática y ciencias.

[5] UNESCO (2004): Las tecnologías de la información y la comunicación en la formación docente. Paris: UNESCO.

[6] La escala de respuestas esta representada por: 4: Casi siempre; 3: Frecuentemente; 2: Algunas veces; 1: Rara vez

[7] La escala de respuesta esta representada por: 4: Casi siempre; 3: Frecuentemente; 2: Algunas veces; 1: Rara vez

(C) Ediciones Universidad de Salamanca 